\title{
Erratum to: HIV Prevention Among Transgender Populations: Knowledge Gaps and Evidence for Action
}

\author{
Tonia Poteat ${ }^{1} \cdot$ Mannat Malik $^{1} \cdot$ Ayden Scheim $^{2} \cdot$ Ayana Elliott $^{3}$
}

Published online: 13 September 2017

(C) Springer Science+Business Media, LLC 2017

Erratum to: Curr HIV/AIDS Rep (2017) 14:141-152

https://doi.org/10.1007/s11904-017-0360-1

The original version of this article unfortunately contained an error in the last sentence of "Biomedical Interventions" section. The change is highlighted in bold below:

None of the transgender women who had blood levels of TDF consistent with taking at least four pills a week seroconverted; and none of the transgender women who seroconverted had detectable levels of TDF in their blood.

The online version of the original article can be found at https://doi.org/ 10.1007/s11904-017-0360-1

Tonia Poteat

tpoteat@jhu.edu

Mannat Malik

mmalik7@jhu.edu

Ayden Scheim

ascheim@uwo.ca

Ayana Elliott

aelliott@coh.org

1 Department of Epidemiology, Johns Hopkins Bloomberg School of Public Health, 615 N. Wolfe Street, E7138, Baltimore, MD 21205, USA

2 Department of Epidemiology and Biostatistics, Schulich School of Medicine and Dentistry, The University of Western Ontario, K201 Kresge Building, London, ON N6B 3J6, Canada

3 Director of Clinical Operations, City of Hope South Pasadena, 209 Fair Oaks Avenue, South Pasadena, CA 91030, USA 